\title{
Elastic Modulus of In-situ Composites of a Liquid Crystalline Polymer and Polycarbonate
}

\author{
QINGHUANG LIN and ALBERT F. YEE \\ Department of Materials Science and Engineering, \\ The University of Michigan, \\ Ann Arbor, Michigan 48109
}

\begin{abstract}
In this paper, we model the elastic modulus of in-situ composite fibers from polymer blends where a fibrous liquid crystalline polymer (LCP) phase is induced by drawing. We propose a composite model to account for the change of the elastic moduli of the reinforcing LCP phase with the draw ratio of the composite fibers. We envisage the LCP phase as a composite of a perfectly oriented chain aggregate and a randomly oriented chain aggregate which are connected in series. We then derive equations for the longitudinal and the transverse elastic moduli of the composite fibers based on the well-known Halpin-Tsai equation and the composite model of the reinforcing LCP phase. Using this approach, we are able to make a number of predictions including the transverse elastic modulus and mechanical anisotropy. Our results show that theoretical predictions of the longitudinal elastic modulus agree fairly well with experimental results for polycarbonate/Vectra composites. The proposed modulus equations will be useful in providing guidelines for fabrication and applications of this new class of polymeric materials.
\end{abstract}

\section{INTRODUCTION}

W e have been interested in developing high-performance, self-reinforced polymer blends where a flexible isotropic polymer is reinforced by a (semi-) rigid liquid crystalline polymer (LCP) (1-6). These blends are usually immiscible due to entropic reasons (7). A unique characteristic of these blends is that it is possible to produce a composite structure with a highly oriented reinforcing fibrous LCP phase in a single step, as opposed to the multiple steps needed in the fabrication of conventional composites. These blends are hence called in-situ composites (8). We and other $(1,4,8-16)$ have shown that, with the addition of only a small amount of LCP, significant improvements in mechanical properties can be achieved. Some key mechanical properties of the resultant in-situ composites are comparable to those of short glass fiber reinforced composites $(4,5)$. Thus in-situ composites offer a promising way to produce high performance polymeric materials at a reduced cost.

One of the methods used to generate an in-situ composite structure - the one used in this work-is co-extrusion of the immisicible LCP and isotropic polymer blend melts followed by drawing, during which the LCP droplets are deformed into fibrils. The drawing process has a paramount effect on the properties of the resulting in-situ composites (3-5). It has been well documented that drawing leads to signifi- cant improvements in certain mechanical properties of such composites. For example, the longitudinal elastic modulus increases steadily with the draw ratio $(4,9,17-21)$. While the causal relationship between drawing and modulus is intuitively obvious, it is desirable to be able to predict the elastic modulus of the in-situ composites with respect to the draw ratio. Such a modeling effort will help determine the optimal processing conditions for this new class of materials.

Unlike in conventional short-fiber reinforced plastics where the reinforcing effect arises from the preformed fibers and their orientation, previous studies have shown that the reinforcement effect in in-situ composites originates from the stretching of the LCP phase in the blend, which increases the aspect ratio of the LCP microfibrils, and the increasing orientation of the LCP chains along the fiber axis during drawing, which enhances the modulus and strength of the LCP microfibrils $(3-5,17,18)$. A new theoretical framework is therefore needed to account for both the molecular orientation and the fibril shape, and their effects on the moduli of the in-situ composites.

Our approach here is based on a composite theory and our understanding of the origin of the reinforcement effect. The LCP phase is envisaged as a composite of a perfectly oriented chain aggregate and a randomly oriented chain aggregate which are connected in series. Equations for the longitudinal and the transverse elastic moduli of the in-situ composite 
fibers are derived on the basis of the well-known Halpin-Tsai equation and the composite model of the reinforcing LCP phase. The theoretical predictions are then compared with the experimental results of the longitudinal modulus of in-situ composite fibers from blends of polycarbonate and a liquid crystalline copoly(ester amide) (Vectra B950). The applications and limitations of the proposed model are also discussed.

\section{MODEL}

In the in-situ composites, the dispersed LCP phase is elongated into a fibrous form during processing. The structure of the in-situ composites is akin to short fiber reinforced composites. We therefore begin with the well-known Halpin-Tsai equation where the elastic modulus of unidirectional short fiber reinforced composites with perfect interfacial adhesion is given as follows (22):

$$
E=E_{m} \frac{1+\xi \eta V_{f}}{1-\eta V_{f}} \quad \eta=\frac{E_{f} / E_{m}-1}{E_{f} / E_{m}+\xi}
$$

For the longitudinal elastic modulus

$$
\xi=2 \frac{L}{D}
$$

while for the transverse elastic modulus

$$
\xi=2
$$

where $E, E_{f}$, and $E_{m}$ are the elastic moduli of the composite, the reinforcing fibrous phase, and the matrix, respectively. Here $L / D$ is the aspect ratio and $V_{f}$ is the volume fraction of the fibrous phase.

It has been demonstrated $(3-5,17,18)$ that both the molecular orientation and the aspect ratio of the reinforcing LCP phase increase with drawing. It follows that the elastic modulus of the LCP phase will also change with drawing. Therefore, the Halpin-Tsai equation cannot be applied directly to the in-situ composites. The objective of this work is to develop two relationships with the draw ratio: one is the relationship between the aspect ratio of the LCP phase and the draw ratio, and the other is the relationship between the elastic modulus of the LCP phase and the draw ratio.

We assume that the deformation, i.e., the draw ratio, of the LCP phase is identical to that of the composite fiber, and that there is no volume change during drawing. It then follows that

$$
\xi=2 \lambda^{3 / 2}
$$

for the longitudinal elastic modulus, where $\lambda$ is the draw ratio of the composite fiber, which is defined as the ratio of the cross-sectional area of the original fiber to that of the drawn fiber.

To account for the change of the elastic modulus of the LCP phase with drawing, we envisage the LCP phase itself as a composite of a perfectly oriented chain aggregate and a randomly oriented chain ag- gregate. The fraction of the perfectly oriented chains $f$ is given by the order parameter of the LCP chains. The fraction of the randomly oriented chains is therefore $(1-f)(23)$. With increasing draw ratio, the fraction of the oriented chains increases at the expense of the random chains. We further propose that the perfectly oriented and the randomly oriented chain aggregates are connected in series (Fig. 1); therefore the iso-stress condition holds (24).

Assuming affine deformation, the order parameter of the LCP phase resulting from an elongational deformation can be written as $(3,25)$ :

$$
f=\frac{2 \lambda^{3}+1}{2\left(\lambda^{3}-1\right)}-\frac{3}{2} \frac{\lambda^{3}}{\left(\lambda^{3}-1\right)^{3 / 2}} \operatorname{Sin}^{-1}\left(\frac{\lambda^{3}-1}{\lambda^{3}}\right)^{1 / 2}
$$

where $\lambda$ is the draw ratio. Thus, the elastic modulus of the LCP phase can be expressed by the following formula based on the composite model:

$$
\begin{aligned}
\frac{1}{E_{f}}= & \frac{f}{E_{c}}+\frac{1-f}{E_{r}} \\
= & \frac{1}{E_{r}}+\left(\frac{1}{E_{c}}-\frac{1}{E_{r}}\right)\left[\frac{2 \lambda^{3}+1}{2\left(\lambda^{3}-1\right)}-\frac{3}{2} \frac{\lambda^{3}}{\left(\lambda^{3}-1\right)^{3 / 2}}\right. \\
& \left.\times \operatorname{Sin}^{-1}\left(\frac{\lambda^{3}-1}{\lambda^{3}}\right)^{1 / 2}\right]
\end{aligned}
$$

where $E_{f}$ is the longitudinal elastic modulus of the reinforcing LCP fibrils. $E_{r}$ is the elastic modulus of the randomly oriented chain aggregate, and $E_{c}$ is the elastic modulus of the perfectly oriented chain aggregate along the chain direction.

Inserting Eqs 2 and 4 into $E q$ 1, we obtain the following equations for the longitudinal elastic modulus of this model composite fiber:

$$
E_{1}=E_{m} \frac{1+2 \lambda^{3 / 2} \eta V_{f}}{1-\eta V_{f}} \quad \eta=\frac{E_{f} / E_{m}-1}{E_{f} / E_{m}+2 \lambda^{3 / 2}}
$$

where

$$
\begin{aligned}
\frac{1}{E_{f}}= & \frac{1}{E_{\tau}}+\left(\frac{1}{E_{c}}-\frac{1}{E_{\tau}}\right)\left[\frac{2 \lambda^{3}+1}{2\left(\lambda^{3}-1\right)}-\frac{3}{2} \frac{\lambda^{3}}{\left(\lambda^{3}-1\right)^{3 / 2}}\right. \\
& \left.\times \operatorname{Sin}^{-1}\left(\frac{\lambda^{3}-1}{\lambda^{3}}\right)^{1 / 2}\right]
\end{aligned}
$$

Similarly, using the series connection of the perfectly oriented aggregate and the randomly oriented aggregate, we obtain the following equations for the transverse elastic modulus:

$$
E_{T}=E_{m} \frac{1+2 \eta V_{f}}{1-\eta V_{f}} \quad \eta=\frac{E_{f}^{1} / E_{m}-1}{E_{f}^{1} / E_{m}+2}
$$




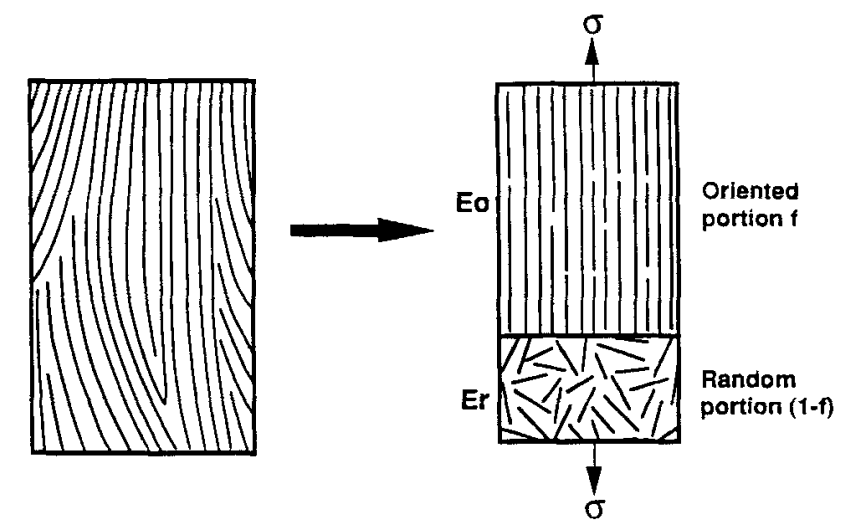

Fig. 1. A composite model for a partially oriented LCP phase.

where

$$
\begin{aligned}
\frac{1}{E_{f}^{\prime}}= & \frac{1}{E_{r}}+\left(\frac{1}{E_{t}}-\frac{1}{E_{r}}\right)\left[\frac{2 \lambda^{3}+1}{2\left(\lambda^{3}-1\right)}-\frac{3}{2} \frac{\lambda^{3}}{\left(\lambda^{3}-1\right)^{3 / 2}}\right. \\
& \left.\times \operatorname{Sin}^{-1}\left(\frac{\lambda^{3}-1}{\lambda^{3}}\right)^{1 / 2}\right]
\end{aligned}
$$

Here $E_{f}$ is the transverse elastic modulus of the reinforcing LCP fibrils, and $E_{t}$ is the elastic modulus of the perfectly oriented chain aggregate perpendicular to the chain direction.

\section{EXPERIMENTAL}

\section{Materials}

The LCP used in this research was Vectra $B 950$ (Hoechst Celanese). It is a random copolymer of three components: $60 \mathrm{~mol} \%$ 2,6-hydroxynaphthoic acid, 20 mol\% terephthalic acid, and $20 \mathrm{~mol} \%$ paraaminophenol. The isotropic thermoplastic matrix was a bisphenol-A polycarbonate (PC) (Lexan 151, GE Plastics).

\section{Sample Preparation}

In-situ composite fibers were fabricated by extrusion of the LCP and polycarbonate blends with a ZSK-30 twin screw extruder (Werner \& Pfleiderer). The extrusion was followed by drawing the extrudate melts directly with a rotating drum winder. The draw ratio was varied by varying the drum's rotational velocity. The detailed procedures are described elsewhere $(4,5)$. Three series of samples were fabricated. One varied in LCP content but with a constant draw ratio of around 11.5; the other two varied in draw ratio but with constant LCP concentrations of 20 and 26.9 volume $\%$, respectively. The draw ratio was determined experimentally from the ratio of the cross-sectional area of the die to that of the extruded composite fiber.

\section{Mechanical Testing}

The longitudinal elastic moduli of the extruded fibers were measured on an Instron Mechanical Tester
(Model 4502). Elongation was determined with the aid of a 0.5 in $50 \%$ extensometer attached directly to the specimen. To reduce stress concentration at the grips, specimens were held in place with rubber pads. All tests were run at a constant crosshead speed of $0.5 \mathrm{~mm} / \mathrm{min}$. All data were acquired by an IBM PC computer and the elastic moduli were calculated by a built-in computer program. Each data point was the average of five measurements.

\section{RESULTS AND DISCUSSION}

The mechanical properties of in-situ composites have been a subject of intensive study. It has been well established that both the elastic modulus and the tensile strength of the in-situ composites increase monotonically with LCP content and draw ratio $(5,9,17-21)$. However, not until recently has the origin of the reinforcement effect been revealed. Attempts have been made to apply the simple rule of mixtures of continuous fiber reinforced composites to the in-situ composites even though in most cases the morphologies of the in-situ composites were far from that of continuous fiber reinforced composites $(1,2,9,10,26-29)$.

Recently some researchers (10.30-32) have pointed out that the aspect ratio of the LCP reinforcing phase could seriously affect the elastic modulus of the in situ composites. By assuming constant values for the elastic moduli of the reinforcing LCP microfibrils and the isotropic matrix, Shin, et al (31), showed that the relative modulus of PET/LCP in-situ composites could double by increasing the draw ratio from 1 to around 100. Unfortunately, the assumption of a constant elastic modulus for the LCP microfibrils is not reasonable because the molecular orientation of the reinforcing LCP phase increases with drawing, and the elastic modulus of the LCP microfibrils is expected to change accordingly. In fact, a careful examination of the literature reveals that the values used for the elastic moduli of the reinforcing LCP fibrils (Vectra A) ranged from 2.2 (29) to $75 \mathrm{GPa}(10)$ with many in between, e.g., 10 (26), 24.6 (9), 26.4 (28), and 70.7 GPa (30). This wide range of LCP modulus values reflects the fact that the elastic modulus of oriented LCPs depends heavily on processing conditions.

In this paper, we not only take into account the variation of the aspect ratio of the LCP phase during drawing, but also consider the effect of drawing on the molecular orientation of the LCP chains, and hence the elastic modulus of the LCP phase, by proposing the composite model for the reinforcing LCP phase as described above. The application of the model and the results are described in the following.

\section{$E_{c}, E_{t}$, and $E_{r}$}

Three non-adjustable parameters have been introduced in the derivation of the equations for the elastic moduli of in-situ composites, i.e., elastic modulus of the perfectly oriented LCP aggregate along the chain direction $E_{c}$, and perpendicular to the chain direc- 
tion $E_{t}$, and that of the randomly oriented aggregate $E_{r}$. In the case of infinitely long polymer chains, $E_{c}$ is the chain modulus, while $E_{r}$ is the modulus of the unoriented LCP. $E_{c}, E_{t}$, and $E_{r}$ are material properties which do not change with processing conditions. There are three ways to obtain values for these three parameters:

First, $E_{c}, E_{t}$, and $E_{r}$ can be acquired by extrapolating the modulus $v$ s, order parameter curve to the limits of perfect orientation and random orientation.

Second, if the chemical composition of the reinforcing phase in question is known, then, in principle, it is possible to compute the elastic moduli of the polymer along the chain direction and the transverse direction by, for example, the lattice dynamics approach (33). The elastic modulus of the randomly oriented polymer can then be calculated according to a composite theory (34).

Third, the chain modulus of oriented polymers can be obtained by in-situ X-ray measurements $(35,36)$. With the assumption of homogeneous stress throughout the test specimen, the crystal chain modulus of a polymer can be determined from the measurements of the lattice strain under a known applied load.

In this paper, we use the first approach to estimate $E_{c}, E_{t}$, and $E_{r}$. The extrapolated moduli are then compared with results from other approaches. Values of $E_{c}$ and $E_{r}$ are obtained by extrapolating an experimentally determined relationship of the elastic modulus of the Vectra B series and order parameter (37), and value of $E_{t}$ is acquired by extrapolating an experimentally determined relationship of the transverse elastic modulus of the Vectra $A$ and $B$ series and draw ratio $(38,39)$. The extrapolation results are $E_{r}=$ $3.8 \mathrm{GPa}, E_{\mathrm{c}}=110 \mathrm{GPa}$, and $E_{t}=2.2 \mathrm{GPa}$, for Vectra B950 LCP.

Compared with values obtained using other approaches, the extrapolated values of the oriented Vectra are quite reasonable. Tashiro and Kobayashi $(33,40)$ have conducted a series of calculations of the theoretical chain moduli of aromatic polyesters and polyamides using the lattice dynamics method. They found that the chain moduli of para-linked aromatic polyesters range from 154 to $178 \mathrm{GPa}$, and those of para-aromatic polyamides are about $182 \mathrm{GPa}$. Due to the incorporation of the naphthalene ring in Vectra 950 , which produces a kink in the chain, its chain modulus is expected to be lower than those of paralinked aromatic polyesters and polyamides. Therefore, the extrapolated value (110 GPa) for the oriented LCP aggregate is quite reasonable. Furthermore, this value corresponds very well with results from $X$-ray measurements by Ward and coworkers (41-43). They have found that, depending on chemical composition. the chain moduli of Vectra liquid crystalline polymers vary from 70 to $130 \mathrm{GPa}$ at $20^{\circ} \mathrm{C}$. The chain modulus of a partially amide-linked liquid crystalline polymer, which is most likely Vectra B, was determined to be around $100 \mathrm{GPa}$ (41). X-ray measurements of a number of oriented polymers showed that values of the elastic modulus perpendicular to the chain direction vary from 2 to $5 \mathrm{GPa}$ (44). The extrapolated values for $E_{t}$ and $E_{r}$ of the LCP fall well within this range.

\section{Theoretical Predictions}

According to Eqs 4 to 7 , the longitudinal and the transverse elastic moduli of the in-situ composite fibers depend on the draw ratio and the reinforcing LCP concentration, in addition to the material properties of the two components. The longitudinal modulus can be calculated using Eqs 4 and 5, and the transverse modulus can be obtained using Eqs 6 and 7 . Figures 2 and 3 show the theoretical predictions of the longitudinal and the transverse elastic moduli for polycarbonate/Vectra composite fibers. Recall that $E_{c}=110 \mathrm{GPa}, E_{t}=2.2 \mathrm{GPa}, E_{r}=3.8 \mathrm{GPa}$ are assumed for the Vectra liquid crystalline polymer. A value of $E_{m}=2.5 \mathrm{GPa}$ is used for the modulus of the polycarbonate matrix in the theoretical predictions (45).

As can be seen from the Figures, the model makes several interesting predictions. Notable among these are the following:

1) Reinforcement can be improved by increasing either the draw ratio or the LCP volume fraction. However, these two factors affect the elastic moduli in two distinctly different fashions: the longitudinal elastic modulus increases steadily with the LCP volume fraction; in contrast, for a given composition, the longitudinal elastic modulus rises sharply toward an asymptotic value with the draw ratio. The draw ratio at which a substantial fraction $(95 \%)$ of the asymptotic value is reached, i.e., the critical draw ratio, increases with the LCP volume fraction. This finding implies that to maximize the reinforcement effect, higher draw ratios are required for composites with higher LCP volume fractions.

2) At higher draw ratios, the longitudinal elastic modulus follows a near-linear relationship with LCP content. This is because at higher draw ratios the aspect ratios of the reinforcing $L C P$ phase become so large that the difference with continuous fibers is insignificant. Therefore the simple rule of mixtures applies. The transverse elastic modulus also follows the rule of mixtures at higher draw ratios.

3) Draw ratio and LCP volume fraction influence the transverse elastic modulus in a manner totally different from that of the longitudinal elastic modulus. In general, the transverse modulus is insensitive to the LCP volume fraction due to the close values of the $E_{t}$ and $E_{m}$. In addition, it drops slightly with the draw ratio.

4) Mechanical anisotropy is clearly evident for the in-situ composite fibers according to the theoretical model. For a constant LCP concentration, the mechanical anisotropy $\left(E_{V} / E_{T}\right)$ increases with the draw ratio because the longitudinal modulus increases with the draw ratio, whereas the transverse modulus decreases (Fig. 4).

5) The theoretical model is able to predict not only the longitudinal modulus but also the transverse 


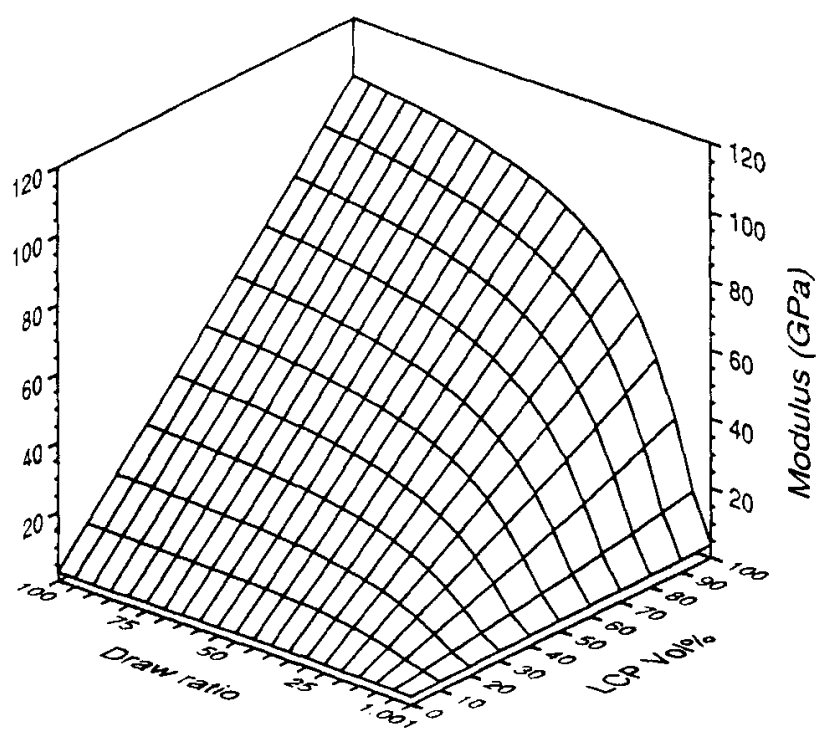

Fig. 2. Theoretical predictions of the longitudinal elastic modulus for polycarbonate/Vectra composite strands. Polycarbonate $E_{m}=2.5 \mathrm{GPa}$, Vectra $E_{c}=110 \mathrm{GPa}$, and $E_{r}=3.8 \mathrm{GPa}$.

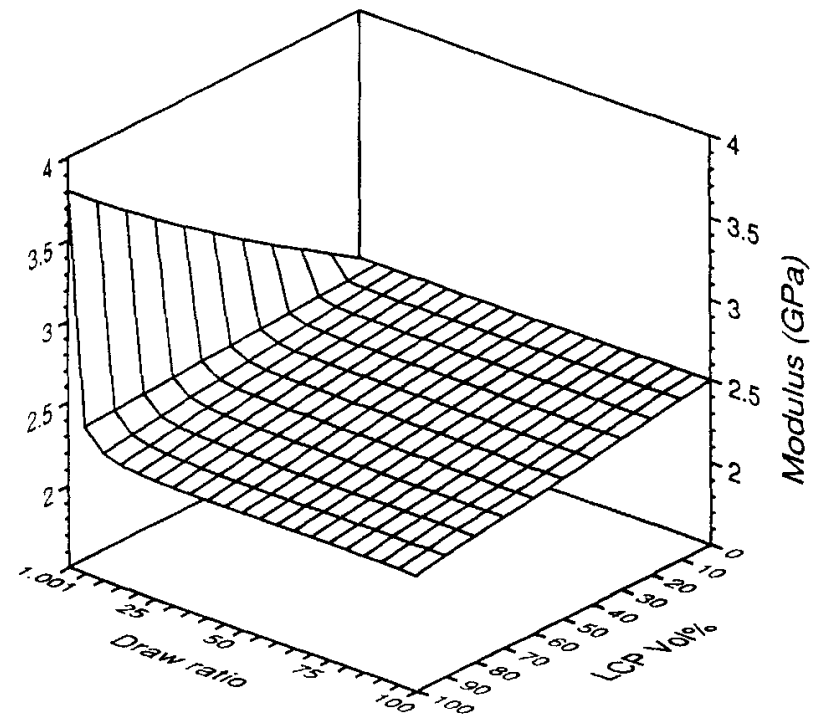

Fig. 3. Theoretical predictions of the transverse elastic modut lus for polycarbonate/Vectra composite strands. Polycarbonate $E_{m}=2.5 \mathrm{GPa}$. Vectra $E_{t}=2.2 \mathrm{GPa}$, and $E_{r}=3.8 \mathrm{GPa}$.

modulus. The prediction of the transverse elastic modulus is of considerable practical significance, because it is very difficult to measure experimentally the transverse elastic modulus of the in-situ composite fibers. Our theoretical approach offers an estimate of this important quantity.

\section{Comparison with Experimental Results}

We now compare the theoretical predictions with experimental results of the polycarbonate/Vectra composite fibers. Since measurements of the transverse elastic modulus of the in-situ composite fibers are very difficult, in the present study we only compare the theoretical predictions and experimental re-

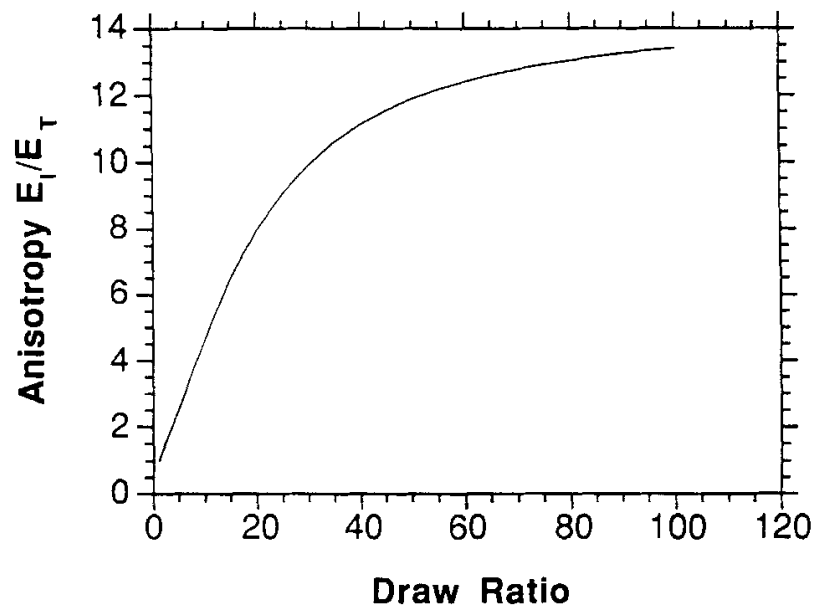

Fig. 4. Mechanical anisotropy as predicted by the proposed model for polycarbonate/Vectra composites with 30 vol ume\% LCP.

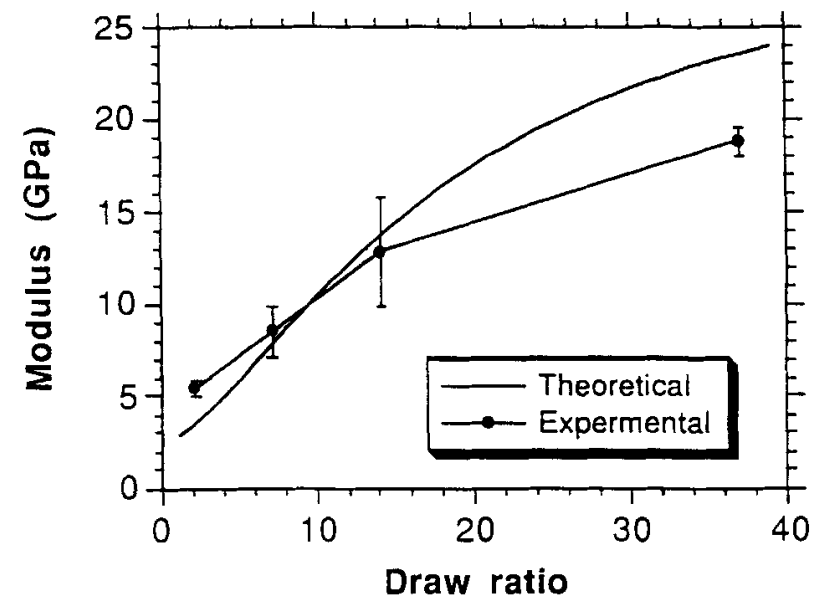

Fig. 5. Comparison of theoretical predictions and experimental results for the longitudinal elastic modulus of polycarbort ate / Vectra composite strands with Polycarbonate $E_{m}=2.5$ $\mathrm{GPa}$, Vectra $\mathrm{E}_{c}=110 \mathrm{GPa}$, and $\mathrm{E}_{r}=3.8 \mathrm{GPa}$. (26.9 volume\% LCP.)

sults of the longitudinal elastic modulus. The comparisons are shown in Figs. 5 to 7 . These Figures clearly demonstrate that theoretical predictions of the longitudinal elastic modulus agree fairly well with the experimental results. In Figs. 5 and 6 , we notice that the best agreement occurs at intermediate draw ratios. Experimental values are lower than the predicted ones at higher draw ratios, but higher than the predicted ones at lower draw ratios. The deviations at higher draw ratios may arise from slippage of the LCP chains, which is not taken into consideration in the derivation of the order parameter. The deviations at lower draw ratios, on the other hand, may suggest that molecular orientation due to shear deformation during extrusion is not negligible at small draw ratios. It is interesting to note that the theoretical predictions correspond reasonably well with the experimental results even at higher LCP contents where the LCP is no longer the discrete phase (Fig. 7). 


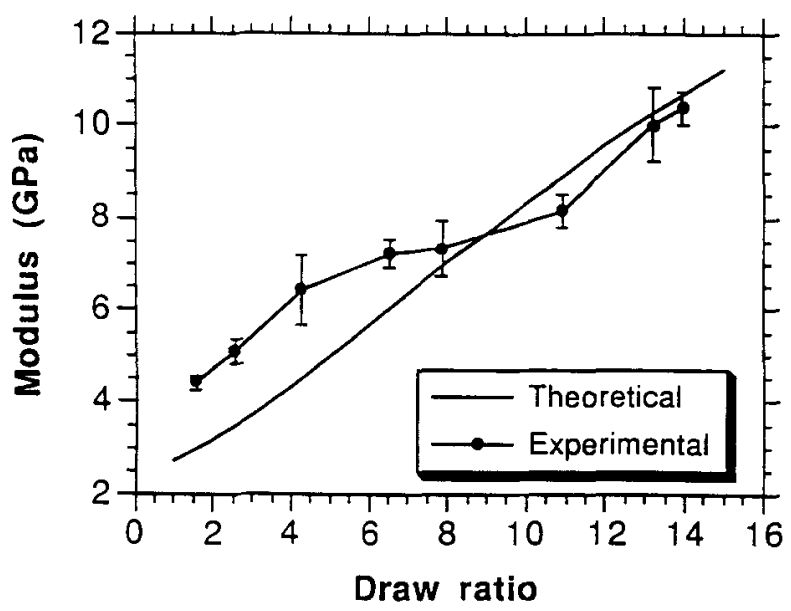

Fig. 6. Comparison of theoretical predictions and experimen tal results for the longitudinal elastic modulus of polycarbonate / Vectra composite strands with Polycarbonate $E_{m}=2.5$ $\mathrm{GPa}$, Vectra $E_{c}=110 \mathrm{GPa}$, and $E_{r}=3.8 \mathrm{GPa}$. (20 volume\% LCP.)

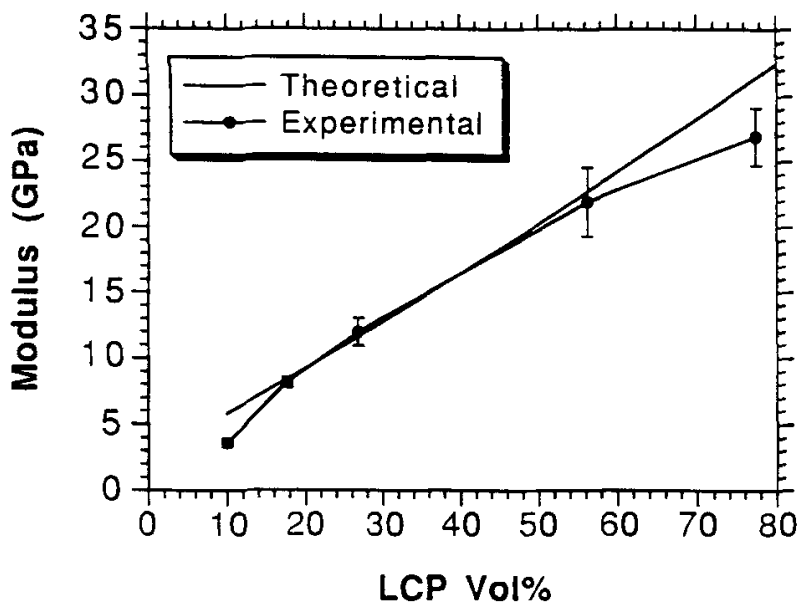

Fig. 7. Comparison of theoretical predictions and experimental results for the longitudinal elastic modulus of polycarbort ate/Vectra composite strands with Polycarbonate $E_{m}=2.5$ $G P a$, Vectra $E_{c}=110 \mathrm{GPa}$, and $E_{r}=3.8 \mathrm{GPa}$. (Draw ratio $=$ 11.5.)

Our previous studies (3-5) showed that drawing of the in-situ composites not only results in the stretching of the LCP phase and the orientation of the LCP chains but also brings about other structural changes. The LCP crystallite size also increases with drawing. Furthermore, some orientation of the polycarbonate matrix will inevitably result from the drawing process. These structural changes can also influence the elastic modulus of the in-situ composite fibers. Obviously, the present model is a simplified one. Nonetheless, the good agreement between the theoretical predictions and the experimental results suggests that the elastic modulus of the in-situ composites is dominated by the aspect ratio and the chain orientation of the reinforcing LCP phase. The crystallite size of the LCP phase as well as the orientation of the polycarbonate matrix have only a minor effect. In fact, since the elastic modulus of oriented LCP fibers is generally several times greater than that of the polycarbonate matrix, the elastic modulus of the in-situ composites is essentially determined by the modulus of the LCP reinforcing phase. Therefore, the orientation of the polycarbonate matrix is not important for the elastic modulus of the overall in-situ composites. This finding in turn supports our previous conclusion about the origin of the reinforcement effect in in-situ composites, i.e., the reinforcement effect in in-situ composites originates from the stretching of the LCP phase and the orientation of the LCP chains (3-5).

\section{Limitations of the Present Model}

As mentioned before, the model proposed in this paper is a simplified one. The following two simplifying assumptions may limit its applications.

1) The deformation of the LCP droplets during processing is assumed to be affine. An ellipsoidal or a fibrous LCP morphology is always supposed to result from drawing. In reality, the morphology of the LCP phase depends on many other factors besides drawing. Even though drawing is one of the key processing parameters, it does not always give rise to a fibrous LCP morphology.

2) The Halpin-Tsai equation assumes that a perfect interface exists between the reinforcing phase and the matrix. However, blends of an LCP with an isotropic polymer have been shown to have fairly weak interfaces $(20,46,47)$. The interfacial adhesion influences the transfer of stress from the polycarbonate matrix to the LCP phase during processing as well as in tensile measurements. Measurements of elastic modulus, however, involve small deformations in the elastic regions. Therefore, the interfacial adhesion is not critical for the elastic modulus of the in-situ composites. But a weak interface can affect the affine assumption, depending on the relative moduli of the two melt phases. The greater the difference, the more important the adhesion is.

We are aware that agreement with experimental results could improve if more complicated models are devised for the LCP phase $(48,49)$. However, in these cases new adjustable parameters would have to be introduced, which is not our intent.

\section{CONCLUSIONS}

A model has been proposed for the elastic modulus of in-situ composite fibers produced by extrusion and subsequent drawing of blends of a liquid crystalline polymer and an isotropic flexible polymer. In the modeling, the aspect ratio of the reinforcing LCP phase is correlated to the draw ratio by assuming an affine deformation scheme. The elastic moduli of the LCP phase are related to the draw ratio through a composite model for the LCP phase itself. The LCP phase is envisaged as a composite of a perfectly oriented chain aggregate and a randomly oriented chain aggregate which are connected in series. Equations for the lon- 
gitudinal and the transverse elastic moduli of the in-situ composites are then derived based on the well-known Halpin-Tsai equation and the composite model of the reinforcing LCP phase.

Using this approach, we are able to make a number of predictions about the effects of the LCP volume fraction and the draw ratio on the moduli of the in-situ composites including the transverse elastic modulus and mechanical anisotropy. Our results show that to maximize the reinforcement effect in the in-situ composites, higher draw ratios are required for composites with higher LCP volume fractions. We further find that theoretical predictions of longitudinal elastic modulus agree fairly well with experimental results for polycarbonate/Vectra composite fibers. The good agreement between the theoretical predictions and the experimental results suggests that the elastic modulus of the in-situ composites is dominated by the aspect ratio of the reinforcing LCP phase and the molecular orientation in the LCP phase.

\section{ACKNOWLEDGMENTS}

We are grateful to Prof. C. L. Choy for pointing out an error in our original manuscript, and for suggesting suitable references.

\section{REFERENCES}

1. B. R. Bassett and A. F. Yee, Polym. Compos., 11, 10 (1990).

2. R. J. Amundsen and A. F. Yee, in Proceedings of the American Society for Composites, Fifth Technical Conference, p. 209, E. Lansing, Mich. (1990).

3. Q. Lin and A. F. Yee, ACS Polym Prepr., 33(1), 298 (1992).

4. Q. Lin and A. F. Yee, SPE ANTEC Tech Papers, 38, 2244 (1992).

5. Q. Lin, J. Y. Jho, and A. F. Yee, Polym. Eng. Sci., 33, 789 (1993).

6. Q. Lin and A. F. Yee, ACS Polym. Prepr., 34(1), 753 (1993)

7. P. J. Flory, Macromolecules, 11(6), 1138 (1978).

8. G. Kiss, Polym. Eng. Sci, 27, 410 (1987).

9. K. G. Blizard, C. Federici, O. Federico, and L. L. Chapoy, Polym. Eng. Sci., 30, 1442 (1990)

10. G. Crevecoeur and G. Groenickx, Polym. Eng. Sci., 30, 532 (1990)

11. B. Y. Shin and I. J. Chung, Polym. J., 21 (1 1), 851 (1989).

12. A. Datta, H. H. Chen, and D. G. Baird, Polymer, 34(4), 759 (1993).

13. R. E. S. Bretas and D. G. Baird, Polymer, 33(24), 5233 (1992).

14. A. Siegmann, A. Dagan, and S. Kenig, Polymer, 26, 1325 (1985).

15. G. Crevecoeur and G. Groeninckx, Bull Soc. Chim. Belg., 99(11-12), 1031 (1990).

16. W. Brostow, Polymer, 31, 979 (1990).

17. C. Carfagna, E. Amendola, L. Nicolais, D. Acierno, O. Francescangeli, B. Yang, and F. Rustichelli, J. Appl. Polym. Sci. 43, 839 (1991).

18. D. Dutta, R. A. Weiss, and K. Kristal, Polym Compos., 13, 394 (1992)
19. T. Sun, D. G. Baird, H. H. Huang, D. S. Done, and G. L. Wilkes, J. Compos. Mater., 25, 788 (1991).

20. B. Y. Shin and I. J. Chung, Polym. Eng. Sci, 30, 13 (1990)

21. A. Ajji, J. Brisson, and Y. Qu, J. Polym Sci., Polym. Phys. Ed., 30, 505 (1992).

22. J. C. Halpin and J. L. Kardos, Polym. Eng. Sci., 16, 344 (1976)

23. R. D. B. Fraser, J. Chem. Phys., 28(6), 1113 (1958).

24. A parallel connection of the perfectly oriented aggregate and the random oriented aggregate did not give satisfactory results.

25. I. M. Ward, Adv. Polym Sci., 66, 81 (1985).

26. P. R. Subramanian and A. I. Isayev, Polymer, 32(11), 1961 (1991)

27. W.-C. Lee, and A. T. DiBenedetto, Polymer. Eng. Sci., 32 400 (1992)

28. A. Kohli, N. Chung, and R. A. Weiss, Polym Eng. Sci. 29, 573 (1989).

29. N. Chapleau. P. J. Carreau, C. Peleteiro, P.-A. Lavoie, and T. M. Malik, Polym. Eng. Sct., 32, 1876 (1992).

30. J. X. Li, M. S. Silverstein, A. Hiltner, and E. Baer, J. Appl Polym Sci, 44, 1531 (1992).

31. B. Y. Shin, S. H. Jang, I. J. Chung, and B. S. Kim, Polym. Eng. Sci., 32, 73 (1992).

32. W.-C. Lee, A. T. DiBenedetto, J. M. Gromek, M. R. Nobile, and D. Acierno, Polym Eng. Sci. 33, 156 (1993).

33. K. Tashiro and M. Kobayashi, Polymer, 32(3), 454 (1991).

34. B. D. Agarwal and L. J. Broutman, Analysis and Performance of Fiber Composites, John Wiley \& Sons, New York (1990).

35. I. Sakurada and K. Kaji, J. Polym Sci., C31, 57 (1970),

36. W. J. Dulmage and L. E. Contois, J. Polym. Sci., 28, 275 (1958).

37. T. S. Chung, J. Polym. Sci., Polym. Phys. Ed, 26, 1549 (1988).

38. C. L. Choy, W. P. Leung, and A. F. Yee, Polymer, 33(8), 1788 (1992)

39. C. L. Choy, personal communication, 1993.

40. K. Tashiro, M. Kobayashi, and H. Tadokoro, Macro molecules, 10, 413 (1977).

41. H. Zhang, G. R. Davies, and I. M. Ward, Polymer, 33(13), 2651 (1992)

42. D. I. Green, A. P. Unwin, G. R. Davies, and I. M. Ward, Polymer, 31, 579 (1990).

43. G. R. Davies and I. M. Ward, in High Modulus Polymers, p. 37, A. E. Zachariades and R. S. Porter, eds., Marcel Dekker, New York (1988)

44. I. Sakurada, T. Ito, and K. Nakumae, J. Polym. Sci., C15, 75 (1966)

45. D. Freitag, U. Grigo, P. R. Mueller, and W. Nouvertne, in Encyclopedia of Polymer Science and Engineering, 2nd Ed., Vol. 11, p. 656, H. F. Mark, N. M. Bikales, C. G. Overberger, and G. Menges, eds. (1988).

46. S. G. James, A. M. Donald, I. S. Miles, L. Mallagh, and W. A. MacDonald, J. Polym. Sci, Polym Phys., 31, 221 (1993).

47. D. Dutta, H. Fruitwala, A. Kohli, and R. A. Weiss, Polym Eng. Sct., 30, 1005 (1990).

48. I. M. Ward, Mechanical Properties of Solid Polymers, 2nd ed., Ch. 10, John Wiley \& Sons, Chichester, England (1983).

49. G. A. Gallagher, R. Jakeways, and I. M. Ward, J. Polym. Sci., Polym. Phys. Ed., 29, 1147 (1991).

Received April 16, 1993 